\title{
Modeling of EHA Module Equipped with Fixed-Displacement Vane Pump
}

\author{
E. Gnesi, J-C. Maré*, J. L. Bordet \\ Parker Hannifin Manufacturing France S.A.S., Vane Pump Division, 14 route du Bois Blanc \\ CS 50601, 18106 Vierzon Cedex, France \\ E-mail: emanuele.gnesi@parker.com,jean-charles.mare@insa-toulouse.fr,JBordet@parker.com \\ * Université de Toulouse; INSA-UPS, Institut Clément Ader, 135 Avenue de Rangueil \\ 31077 Toulouse Cedex, France
}

\begin{abstract}
The present communication deals with the development of new EHA modules for injection molding machines, which involve industrial motor power drive, brushless motor and vane pump for downsizing and energy saving when actuators are used in sequence. Once introduced the evolution in actuation for such applications, the interest of EHA architecture is pointed up. In a second part, a simple control model is developed, highlighting explicitly the functional and parasitic effects for the different mode of use: motor speed control, cylinder pressure control and cylinder position control. The last part is dedicated to the virtual prototyping with special focus on energy losses within the module. Model structures and their implementation are proposed for pump frictions and internal leakages that use standard components of the LMSAMESim libraries. The influence of speed and pressure are introduced either through look-up tables or simple parametric model which parameters are identified from real experiments.
\end{abstract}

Keywords: Actuator, EHA, energy saving, efficiency, injection molding machine, vane pump, AMESim

\section{Introduction}

The paper deals with the model based design of an electrohydrostatic actuator (EHA) module for industrial applications, with special focus on modeling and simulation. The last years growing interest to environmental issues has turned companies policy's attention to research of more ecofriendly solutions with lower environmental impact and energy saving. Thus the energy consumption, the $\mathrm{CO}_{2}$ and acoustic emissions have become essential requirements, in addition to functionality and productivity demands, that the industrial engineering has to take into account during conceptual and preliminary phases.

Within the framework of environment respect, a significant improvement has been put concerning the actuation technology. Initially hydraulically-powered and valvecontrolled, the actuators have been or are being replaced by electrically supplied, power-on-demand solutions, thanks to the increasing maturity of high power electronics and the progress of rare earth electric motors and their controls. An example is represented by the aerospace industry which in last decade has dedicated itself to development of greener aircrafts: in the commercial transport the recent A380 and the Boeing 787 are considered the first "more electric aircrafts" due to adoption of power-by-wire actuators such as EHAs, electro-backup hydrostatic actuators (EBHAs) and electromechanical actuators (EMAs) principally in backup mode for primary and secondary flight controls, in place of the conventional servohydraulic actuators (SHAs) [1].

The trend has affected the stationary machinery in the industrial automation too. It includes the drive technology for motion control in the injection molding machines. As this is one of the highest energy consuming sector, the design studies do focus more and more on energy efficiency improvement. The injection molding machine is a cyclical machine producing plastic or rubber parts: it generally consists of some axes (at least six) moved sequentially by a drive module, the machine's heart, which was usually in the conventional design operated using a centralized pressure/flow control. Today the demands on drive systems and control are pressing to increase service pressure, velocities and repeatability over a long period of time. This enables increasing productivity while saving energy and reducing noise and maintenance costs [2], [3].

All these requirements have led the drive technology to evolve by increasing the interest for the electromechanical and electrohydrostatic modules.

Common practice in the past was the employment of hydraulically driven injection molding machines where the 
axes' control was achieved through proportional valves supplied at a constant pressure. In the nineties, high dynamics response valves with load-sensing features were introduced (fig. 1). The electrohydraulic drives and controls manufactures development started in those years and, for the 2000 's decade, a step forward was achieved by using pressure/flow variable displacement pump controls (fig. 1): the new solution was based on a constant-speed electric motor driving a variable-displacement pump which represented a favorable alternative to valve controlled drives towards reduction of energy losses [4]. Since theses years, the electromechanical machines popularity has been increasing, in particular in the Japanese manufactures. The electromechanical module is typically constituted by an AC servomotor controlled by a frequency converter and driving the translating load through a ball-screw (fig. 1). For an average production cycle, this design can potentially save around $30 \%$ energy compared with the most efficient hydraulic machines due to improved efficiency of electric drive modules. Shorter cycle time and higher accuracy are obtained with simpler control [3]. The drawbacks of this technology mainly concerns cost (about 20-30\% higher than comparable hydraulically driven machines) [4], low capability to dispose the heat generated by the energy losses, high kinetic energy of rotating parts and low tolerance to jamming of mechanical components. This last fault involves a frequent need of maintenance and further cost increase [5].

Recently the demand for drive solutions with higher force density has again pushed to the fore the electrohydraulic ones. In particular the interest concerns the EHA module concept of speed-controlled fixed-displacement pump driven by a permanent magnet synchronous machine with its inverter (fig. 1). By combining the advantages of power-bywire (energy saving) and hydraulics (fluid as a heat conveyor) without requiring a motor power drive per axis, this design makes the injection molding machines more competitive than before [6]. Compared with constant-speed variable-displacement pump, it reveals its advantages of drastic reduction of energy consumption, especially in processes characterized by long phases with no or little oil flow [3]. Therefore, it appears as the principal competitor of the electromechanical modules when accuracy, reliability, flexibility and noise are considered. In particular, maintenance costs are significantly cut as it is cheaper to replace hydraulic seals than ball-screws and bearings.

Several studies have been achieved concerning possible EHA module configurations, just mention modeling of variable-speed electric motor driving gear pump [7] and [8], and solutions are already existing for various application fields as for light industrial and domestic one [9]. Other configurations constituted by variable-speed electric motor driving fixed-displacement axial piston pump that are present on global market as the designs of Liebherr Aerospace (bent axis) or Messier-Bugatti (in-line) for the recent Airbus A380 [10].

In this communication an innovative Parker solution of EHA module is proposed that associates an inverter, a brushless DC motor and a fixed-displacement low-noise vane pump.

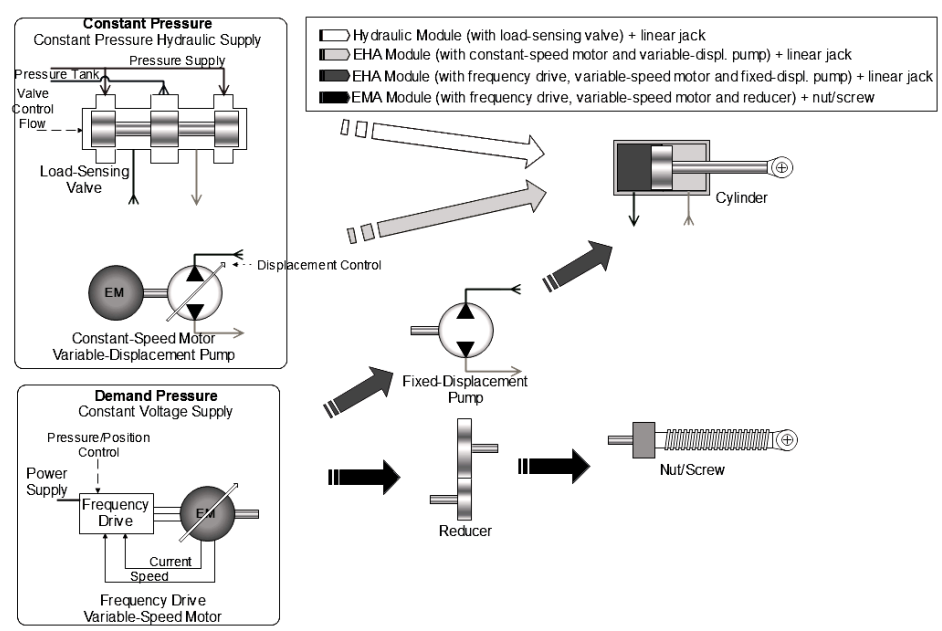

Figure 1: Architecture of modules: hydraulic, EHA and EMA

Depending on the phase of the molding cycle, injection molding machines require the module to be closed-loop controlled either in position, speed or pressure.

The EHA module integrates the advantages of both conventional technologies: easiness of integration (compactness, simplicity), reduced maintenance cost (very few rolling components), resistance to harsh conditions, high power density of hydraulics and high energy efficiency of control electrics of power, high speed response, low noise level, flexibility and easiness of vector control. Parker proposes to immerse the motor-pump monobloc unit inside oil tank. This improves motor cooling and pump suction and reduces the noise level. Figure 2 shows an example of NX Series servomotor and T7 Series vane pump constituting the motor-pump unit of EHA module.

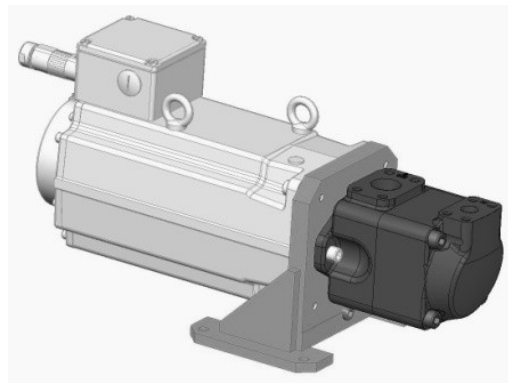

Figure 2: Example of Motor-pump unit of EHA module with $N X$ Series servomotor and T7 Series vane pump

Recent fixed-displacement Parker vane pumps are wellknown for their high performances (operating pressure up to 280 bars in a speed range from 600 to $3600 \mathrm{rpm}$ ), high efficiency, low noise levels, low ripple pressure, good compactness, mounting flexibility and good reliability. The plenty of possible configuration (workable thanks to cartridge concept) makes EHA module versatile and adapted to market requirements.

Section 2 of this paper describes the reference architecture of EHA module while in section 3 preliminary analysis is carried out by developing functional and architectural analytical models (paragraphs 3.1 and 3.2). In paragraph 3.3 control synthesis of the three types of closed loop operation 
(position, pressure and speed) is addressed in a formal way. Paragraph 3.4 concerns the evaluation of parasitic effects on open loop natural dynamics. In section 4 a detail vane pump model with characterization of frictional torques and internal leakages is developed through virtual prototyping in LMSAMESim environment. The non-linear phenomena are also parameterized by getting parametric linear models useful in the control unit design phase of EHA module. Section 5 concerns conclusions and future work planning.

\section{Reference architecture of EHA module}

A reference architecture is identified and showed in fig. 3: a frequency drive (control electronic unit and motor power unit), a variable speed electric motor (brushless DC Motor, BLDC), a fixed displacement pump (vane pump) and a linear hydraulic jack. The control electronic unit performs closed-loop on either rod position or on cylinder pressure. The motor power unit operates in closed-loop on motor shaft speed and an inner closed-loop motor torque control. The external position controller provides the command value for the cylinder pressure controller and its output represents the command for the motor speed control loop. The last one forms then the input for the motor torque loop.

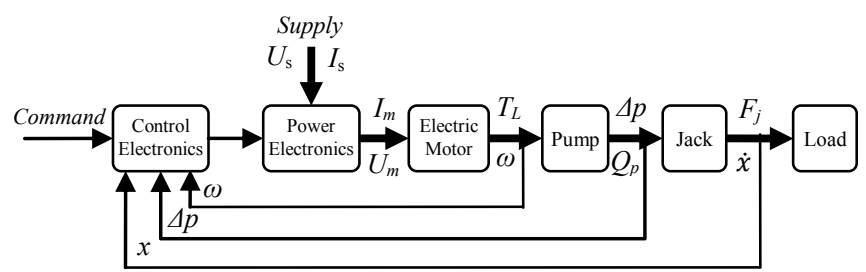

Figure 3: EHA reference architecture schematic diagram

In reference to fig. 3: $x$ is the rod position, $\Delta p$ is the cylinder pressure, $\omega$ is the motor shaft speed, $U_{s}$ and $I_{s}$ are respectively the supply voltage and current, $U_{m}$ and $I_{m}$ the electric motor voltage and current, $T_{L}$ is the load torque acting on the motor shaft, $Q_{p}$ is the output volume flow rate generated by pump, $F_{j}$ is the external load force acting on jack rod and $\dot{x}$ is the translational rod speed.

\section{Model-Based Design}

The scientific approach proposed to develop the research subject, is characterized by a preliminary design based on analytical models (Model-based design) and on virtual prototypes at subsystem level. The EHA study is complex because it is multi-domain, multi-criteria and strong coupling between domains (reflected inertia of the rotor and thermal issues). Multi-domain feature is about to the presence of several physical phenomena coexistent and to be taken into account: a transversal vision is required to work with solid/fluid mechanics, power electronics, electrotechnics, thermal exchanges, control, etc. It has also multi-criteria features: requirements to be fulfilled, are about power capacity, energy consumption, reliability, natural dynamics, control performance, etc.

In detail, a preliminary analysis has been carried out by means of an analytical model at architectural level, obtained by reducing a more detailed model at functional level to a simple ordinary differential equations' system (ODE). It permits to: understand the constraints between variables, the fundamental parameters effects on performance, all aspects which characterize the actuator physical operation principle and address control synthesis in a formal way. Successfully the analysis has been concerned with the advanced model that evaluates the parasitic effects associated to each component: open-loop transfer functions have been analyzed to provide the designer with key relationships between open-loop performance and parasitic effects. Virtual prototypes have been developed in LMS-AMESim environment to validate the theoretical results got from the linear analysis.

\subsection{System level modeling}

The advanced model exposed in this paragraph is represented by a linear functional model that describes the EHA module dynamics with the major parasitic effects. It is the starting point to get the simplified model that will be exposed in 3.2. With reference to fig. 3, the equations relative to electric motor, vane pump and hydraulic jack are introduced.

The BLDC is described by the mathematical equations concerning the Motor Electric Equation and the Momentum Balance referred to motor shaft axis [11]:

$$
\left\{\begin{array}{l}
U_{m}=K_{m} \omega+R I_{m}+L \frac{d I_{m}}{d t} \\
T_{L}=K_{m} I_{m}-J_{m} \dot{\omega}-T_{f m}
\end{array}\right.
$$

where $K_{m}$ is the back-electromotive force coefficient, $R$ is the motor winding resistance, $L$ is the motor winding inductance, $J_{m}$ is the motor inertia and $T_{f m}$ is the frictional motor torque.

By observing the eq.s (1), first terms of second members represent the functional contributions while the remainders represent the parasitic contributions. In order to get the eq.s (1), the following assumptions have been added:

- The frictional torques of motor are considered linear functions of motor shaft speed

$$
T_{f m}=B_{m} \omega
$$

where $B_{m}$ is the motor viscous friction coefficient.

The vane pump dynamics is described by the Flow Conservation Equation, written in reference to Control Volume and the Momentum Balance referred to pump shaft axis:

$$
\left\{\begin{array}{c}
Q_{p}=V_{0} \omega-Q_{l p}-\frac{V_{p}}{\beta} \Delta \dot{p} \\
V_{0} \Delta p=T_{L}-J_{p} \dot{\omega}-T_{f p}
\end{array}\right.
$$

where $V_{0}$ is the pump displacement, $V_{p}$ is the pump volume, $\beta$ is the apparent Bulk modulus of the fluid, $J_{p}$ is the pump 
rotor inertia while the variable $Q_{l p}$ is the internal pump leakage flow and $T_{f p}$ is the frictional pump torque.

Similarly to eq.s (1), first terms of second members represent the functional contributions while the remainders represent the parasitic contributions. In order to get the eq.s (3), the following assumptions have been added:

- The Control Volume is considered rigid (no wall deformations)

- The flow conditions are assumed laminar (all mating pump clearances are made small, leading to laminar flow conditions, [12]). The internal pump leakage is so a linear function of pressure drop:

$$
Q_{l p}=a_{p} \Delta p
$$

where $a_{p}$ is the internal pump leakage coefficient.

- The frictional torques of pump are considered linear functions of motor shaft speed:

$$
T_{f p}=B_{p} \omega
$$

where $B_{p}$ is the pump viscous friction coefficient.

The hydraulic conduit, that links pump and jack, has to be taken into account because its length can reach several meters in plastic injection machines and the flow variations due to fluid compressibility can be relevant. By applying the Flow Conservation Equation for the Control Volume and by adding an empiric equation that describes the pressure drop dependent on laminar/turbulent flow type [12], it results:

$$
\left\{\begin{array}{c}
Q_{j}=Q_{p}-\frac{V_{c}}{\beta} \Delta \dot{p}_{c} \\
\Delta p_{c}=\lambda(R e) \frac{L_{c}}{D_{c}} \frac{\rho\left(\frac{Q_{p}}{A_{c}}\right)^{2}}{2}
\end{array}\right.
$$

where $V_{c}$ is the volume of pipe, $\lambda(R e)$ is the friction factor (that depends on Reynolds number $R e$ and pipe roughness), $L_{c}$ is the pipe length, $D_{c}$ is the pipe diameter and $A_{c}$ is the transversal section pipe area. The variable $Q_{j}$ is the flow rate coming into jack chamber, $\Delta p_{c}$ is the pressure drop between the extremes of pipe and $\rho$ is the fluid density.

In order to get the eq.s (6), the following assumptions have been added:

- The Control Volume is considered rigid (no wall deformations). In practice its compliance is implicitly considered in the apparent Bulk modulus of the fluid

- Leakage flow rates due to presence of hydraulic valves are neglected
The hydraulic jack is asymmetrical (the jack chambers symmetry is not needed because injection molding machines require unidirectional forces to be generated by each axis unit and they consider the motion inversion only as a return to initial condition). However, for linear analysis it is considered as an equivalent symmetrical cylinder [13]. The Mass Conservation equation applied to jack chamber and the Second Newton's Law equation applied to rod are:

$$
\left\{\begin{array}{c}
S \dot{x}=Q_{j}-Q_{l j}-\frac{V_{j}}{\beta} \Delta \dot{p} \\
F_{j}=S \Delta p-M \ddot{x}-F_{f j}
\end{array}\right.
$$

where $S$ is the active area of the piston,,$V_{j}$ is the jack chamber volume, $M$ is the total mass of piston and load. The variable $Q_{l j}$ is the jack chamber leakage and $F_{f j}$ is the piston/cylinder frictional force.

First terms of second members of the eq.s (7) represent the functional contributions while the remainders represent the parasitic contributions. In order to get the eq.s (7), the following assumptions have been added:

- The jack chamber volume is considered rigid (no wall deformations)

- The fluid conditions are assumed laminar and so the internal leakage is so a linear function of pressure drop [12]:

$$
Q_{l j}=a_{j} \Delta p
$$

where $a_{j}$ is the cross-chamber leakage coefficient.

- The piston/cylinder friction is assumed to be a linear function of rod speed:

$$
F_{f j}=f \dot{x}
$$

where $f$ is the piston/cylinder viscous friction coefficient.

Concerning the power electronics, the motor voltage and current are correlated to supply voltage and current through the simplified following expressions:

$$
\left\{\begin{array}{l}
U_{m}=\alpha U_{s} \\
I_{m}=\frac{1}{\alpha} I_{s}
\end{array}\right.
$$

where $\alpha$ is power modulation factor and the assumption of ideal power electronics is made (no voltage drop through closed switch and no current leak through opened switch).

Before evaluating the parasitic effects on actuator performance, a simplification of the equations' system has been carried out in next paragraph in order to: understand the constraints between variables, the fundamental parameters effects on performance and all aspects which 
characterize the actuator physical operation principle and address control synthesis in a formal way.

\subsection{Architectural model}

In first design phase, a preliminary analysis at "architectural level" has been carried out in order to evaluate the EHA module physical operation principle without taking into account parasitic effects. Under these assumptions, the eq.s (1) of the electric motor reduce to:

$$
\left\{\begin{array}{c}
U_{m}=K_{m} \omega \\
T_{L}=K_{m} I_{m}
\end{array}\right.
$$

The eq.s (3) of pump are reduced to:

$$
\left\{\begin{array}{c}
Q_{p}=V_{0} \omega \\
V_{0} \Delta p=T_{L}
\end{array}\right.
$$

The eq.s (6) of hydraulic lines are reduced to:

$$
Q_{j}=Q_{p}
$$

The eq.s (7) of hydraulic jack are reduced to:

$$
\left\{\begin{array}{c}
Q_{j}=S \dot{x} \\
F_{j}=S \Delta p
\end{array}\right.
$$

This enables relating the steady-state operation with fundamental parameters to be defined during the early design phase, in particular for sizing and component reference selection: $K_{m}, V_{0}$ and $S$.

\subsection{Control synthesis: system performance in closed- loop operation}

The controller is designed for three types of closed loop operation (position, pressure and speed). A study is conducted on major requirements (accuracy, rapidity and stability) the controlled dynamical system has to satisfy. The controllers taken into account are: proportional (P), proportional integral (PI), proportional derivative (PD) and proportional integral derivative (PID) because of their availability in industrial motor drives. In order to accomplish correctly its functions, the controlled system has to be developed assuring some requirements on both steadystate and transient condition. In time domain, the closed loop system response is analyzed by means of accuracy and rapidity proprieties while in frequency domain by means of the stability propriety [14]: the accuracy is evaluated through static error calculation $\varepsilon_{s}$, the rapidity through response time $t_{s}$ and the asymptotic stability through the poles locations in the Complex Plane (Real, Imaginary). All controllers are initially considered proportional that aims at providing a performance reference and at pointing out the effort in controller design to be done for meeting the performance requirements. An analysis more accurate has to be conducted about system robust stability [15].
Concerning the rod position control, the load position $(x)$ signal is achieved by a voltage control signal $U_{m}$ : it is obtained by integration of rod speed $\dot{x}$ in the eq. (14):

$$
x(s)=\frac{V_{0}}{S K_{m} s} U_{m}(s)
$$

where $\frac{V_{0}}{S K_{m}}$ represents the ideal speed gain of the process for load position control and $s$ is the Laplace variable.

Concerning the cylinder pressure control synthesis, the pressure $(\Delta p)$ is varied by a motor current control signal $I_{m}$, leading to:

$$
\Delta p(s)=\frac{K_{m}}{V_{0}} I_{m}(s)
$$

where $\frac{K_{m}}{V_{0}}$ is the ideal static gain of the process for pressure control.

Concerning the motor shaft speed control synthesis, the angular velocity $\omega$ signal is varied by a voltage control signal $U_{m}$ leading to:

$$
\omega=\frac{1}{K_{m}} U_{m}
$$

where $\frac{1}{K_{m}}$ is the ideal static gain of the process for motor speed control.

Finally the EHA actuation system for position control is ideally once integrator in open loop while it is only a pure gain for pressure and speed control.

\subsection{Natural Dynamics Analysis}

The analysis executed in last paragraph 3.3, has permitted to provide the designer with key relationships between closed loop performance and fundamental parameters. Next step concerns the addition of the parasitic parameters and their modification effects on natural system dynamics. The functional model described in 3.1, has so been analyzed through its open-loop transfer functions characterization. In order to simplify the treatise, the following assumptions are made:

- The mechanical transmission between motor and pump is considered perfectly rigid: the motor and pump inertia and frictional torques have so been merged as single inertia and friction torque

- The pump volume is considered small making the compressibility flow negligible on the actuator behaviour

- The pressure drop in the pipe between pump and jack is neglected and so the output pump flow rate corresponds to input jack chamber flow rate 
- There is no leakage at jack level due to the presence of seals

- a separation in frequency domain has been fulfilled by deciding to reproduce only the lowest natural frequencies, lower than $600 \mathrm{~Hz}$. So the motor currents loop dynamics have been neglected

Consequently motor inductance and resistance were removed from the model. The modelled motor electromagnetic torque $T_{m}$ is therefore assumed to be coincident with the torque demand $T_{m}^{*}$ :

$$
T_{m}=T_{m}^{*}
$$

A representation in state-space domain has been used in order to reduce the functional model to four first order equations in the four variables: rod position, rod speed, cylinder pressure and motor shaft speed.

$\dot{X}=\left[\begin{array}{cccc}0 & 1 & 0 & 0 \\ 0 & -\frac{f}{M} & \frac{S}{M} & 0 \\ 0 & -\frac{\beta S}{V} & -\frac{\beta a_{p}}{V} & \frac{\beta V_{0}}{V} \\ 0 & 0 & -\frac{V_{0}}{J} & -\frac{B}{J}\end{array}\right] X+\left[\begin{array}{cc}0 & 0 \\ 0 & -\frac{1}{M} \\ 0 & 0 \\ \frac{1}{J} & 0\end{array}\right] u$

where $B$ is the sum of motor and pump viscous coefficient, $V$ is the sum of pipe and jack chamber volume and $J$ is the sum of motor and pump inertia.

The derivative state vector $\dot{X}$, the state vector $X$ and the input system vector $u$ are respectively given by:

$$
\dot{X}=\left[\begin{array}{c}
\dot{x} \\
\ddot{x} \\
\Delta \dot{p} \\
\dot{\omega}
\end{array}\right] ; X=\left[\begin{array}{c}
x \\
\dot{x} \\
\Delta p \\
\omega
\end{array}\right] ; u=\left[\begin{array}{c}
T_{m}^{*} \\
F_{j}
\end{array}\right]
$$

leading to the state space model of eq. (19). This permits getting the natural frequencies and to associate them with the hydraulic and hydromechanical modes.

\subsubsection{Rod position transfer function}

The open-loop transfer function, which describes the rod position dynamics in function of motor shaft speed control signal and of jack force disturbance signal, has been got by reducing the system (19). It results:

$$
x(s)=G_{\omega}^{x}(s) \omega(s)+G_{F}^{x}(s) F_{j}(s)
$$

with $G_{\omega}^{x}(s)=\frac{\frac{\beta V_{0} S}{V M}}{s\left[s^{2}+s\left(\frac{\beta a_{p}}{V}+\frac{f}{M}\right)+\frac{\beta S^{2}}{V M}\left(\frac{a_{p} f}{s^{2}}+1\right)\right]}$

$$
G_{F}^{x}(s)=\frac{-\frac{1}{M}\left(s+\frac{\beta a_{p}}{V}\right)}{s\left[s^{2}+s\left(\frac{\beta a_{p}}{V}+\frac{f}{M}\right)+\frac{\beta S^{2}}{V M}\left(\frac{a_{p} f}{s^{2}}+1\right)\right]}
$$

It is possible to simplify the expressions by adding the following hypothesis:

- The ratio $\frac{a_{p} f}{S^{2}}$ is negligible compared to unit [12]: $\frac{a_{p} f}{S^{2}} \ll 1$

As the denominator in common between the two transfer functions, $D G^{x}(s)$ reduces to:

$$
D G^{x}(s)=s\left[s^{2}+s\left(\frac{\beta a_{p}}{V}+\frac{f}{M}\right)+\frac{\beta S^{2}}{V M}\right]
$$

where the pair of poles is characterized by an hydraulic undamped natural pulsation $\omega_{h}$ and damping ratio $\xi_{h}$ respectively equal to:

$$
\begin{gathered}
\omega_{h}=\sqrt{\frac{\beta S^{2}}{V M}} \\
\xi_{h}=\frac{a_{p}}{2 S} \sqrt{\frac{\beta M}{V}}+\frac{f}{2 S} \sqrt{\frac{V}{\beta M}}
\end{gathered}
$$

In order to increase hydraulic mode natural frequency $f_{h}=\frac{\omega_{h}}{2 \pi}$, it is necessary: to increase the compressibility coefficient $\beta$, the jack piston surface $S$ and to reduce the volume $V$ and the total mass $M$. The natural frequency increase permits to reduce rise time $\left(\propto \frac{1}{\omega_{h}}\right)$ and response time $\left(\propto \frac{1}{\xi_{h} \omega_{h}}\right)$.

In order to increase $\xi_{h}$, it is necessary to increase both $a_{p}$ and $f$ : it permits to reduce the maximum peak of rod position temporal response and to reduce response time $\left(\propto \frac{1}{\xi_{h} \omega_{h}}\right)$. The increase of $a_{p}$ is possible for example by means of a voluntary leakage but paying attention to reduction of volumetric efficiency and increase of tracking and load disturbance error.

In steady-state condition the eq. (21) is reduced to eq. (24) by means of assumption $\frac{a_{p} f}{s^{2}} \ll 1$ :

$$
x(s)=\frac{V_{0}}{S S} \omega(s)-\frac{a_{p}}{S^{2} S} F_{j}(s)
$$

On the one hand the pump leakage increase, through the coefficient $a_{p}$, improves temporal response rapidity through the increase of $\xi_{h}$. On the other hand it makes worse the accuracy (static error) in confront to a motor shaft speed signal due to increase of disturbance contribution associated to jack force signal.

\subsubsection{Cylinder pressure transfer function}

In the same manner as for position control, the open-loop transfer function which describes the cylinder pressure dynamics in function of motor torque signal and of rod 
speed disturbance signal has been got by reducing the system (19) and by getting the expression:

$$
\begin{array}{r}
\Delta p(s)=G_{T_{m}}^{\Delta p}(s) T_{m}(s)+G_{\dot{x}}^{\Delta p}(s) \dot{x}(s) \\
\text { with } G_{T_{m}}^{\Delta p}(s)=\frac{\frac{\beta V_{0}}{V J}}{\left(s^{2}+s\left(\frac{B}{J}+\frac{\beta a_{p}}{V}\right)+\frac{\beta V_{0}^{2}}{V J}\left(1+\frac{a_{p} B}{V_{0}^{2}}\right)\right)} \\
G_{\dot{x}}^{\Delta p}(s)=\frac{-\frac{\beta S}{V}\left(s+\frac{B}{J}\right)}{\left(s^{2}+s\left(\frac{B}{J}+\frac{\beta a_{p}}{V}\right)+\frac{\beta V_{0}^{2}}{V J}\left(1+\frac{a_{p} B}{V_{0}^{2}}\right)\right)}
\end{array}
$$

It is possible to simplify the expressions by adding the following hypothesis:

- The ratio $\frac{a_{p} B}{V_{0}{ }^{2}}$ is negligible compared to unit [12]:

$$
\frac{a_{p} B}{V_{0}{ }^{2}} \ll 1
$$

As the denominator in common between the two transfer functions, $D G^{\Delta p}(s)$, reduces to:

$$
D G^{\Delta p}(s)=\left(s^{2}+s\left(\frac{B}{J}+\frac{\beta a_{p}}{V}\right)+\frac{\beta V_{0}^{2}}{V J}\right)
$$

where the pair of poles is characterized by an hydromechanical undamped natural pulsation $\omega_{h m}$ and damping ratio $\xi_{h m}$ respectively equal to:

$$
\begin{gathered}
\omega_{h m}=\sqrt{\frac{\beta V_{0}^{2}}{V J}} \\
\xi_{h m}=\frac{a_{p}}{2 V_{0}} \sqrt{\frac{\beta J}{V}}+\frac{B}{2 V_{0}} \sqrt{\frac{V}{\beta J}}
\end{gathered}
$$

In order to increase the hydromechanical mode natural frequency $f_{h m}=\frac{\omega_{h m}}{2 \pi}$, it is necessary: to increase the compressibility coefficient $\beta$, the pump displacement $V_{0}$ and to reduce the volume $V$ and the motor-pump inertia $J$. The natural frequency increase permits to reduce rise time and response time.

In order to increase $\xi_{h m}$, it is necessary to increase both $a_{p}$ and $B$ : it permits to reduce the maximum peak of pressure temporal response and to reduce response time.

In steady-state condition the eq. (25) is reduced to eq. (28) by means of assumption $\frac{a_{p} B}{V_{0}{ }^{2}} \ll 1$ :

$$
\Delta p(s)=\frac{1}{V_{0}} T_{m}(s)-\frac{S B}{V_{0}^{2}} \dot{x}(s)
$$

If on the one hand the $B$ viscous friction increase improves temporal response rapidity through the increase of $\xi_{h m}$, on the other hand it makes worse the accuracy (static error) in confront to a motor torque signal due to increase of disturbance contribution associated to rod speed signal.
The theoretical results got by linear analysis until this time, have been validated through simulation tests performed in AMESim environment consisting of a simple model stimulated with different inputs magnitude and in which the several parasitic phenomena have been added progressively.

\section{Detail model: vane pump}

The limitations of the linear approach proposed in 3.4.1 and 3.4.2, provide results not completed realistic. The motivation depends on fact that the parasitic phenomena modeling is not sufficiently representative of all effects correlated to them. Concerning the motor-pump frictions, the viscous coefficient $B$ represents the simplest model to describe the frictions as function of speed. For getting higher accuracy, a more detailed friction model could be expressed as follows:

$$
T_{f}(\omega)=\left[T_{c}+\left(T_{s}-T_{c}\right) e^{-\frac{|\omega|}{\alpha_{k}}}+B|\omega|\right] \operatorname{sign}(\omega)
$$

where $T_{c}$ is the Coulomb friction, $\left(T_{s}-T_{c}\right) e^{-\frac{|\omega|}{\alpha_{k}}}$ is the Stribeck friction and $B|\omega|$ the viscous friction [16]. The friction contribution due to pressure drop could be relevant too. Same considerations can be done about the Bulk modulus $\beta$ : its effective value varies in function of pressure and temperature and it depends also on: presence of bubbles of free gas present inside fluid and deformability of the hydraulic components. The pump leakages are variable in function of operation conditions (pressure, pump speed and temperature) too and a constant coefficient $a_{p}$ results limitative to characterize adequately the phenomena.

In order to improve the EHA module analysis, it is so necessary to evaluate the non-linearities that have not been taken into account in the architectural and functional models. The progressive improvement of the EHA module modeling concerns the evaluation of major non-linearities effects that may alter performance. Therefore a detail model has to be developed, for each component constituting the module, that permits analyzing phenomena in a more realistic way. At this modeling level, a virtual prototype has been developed.

In the present work, the Parker vane pump dynamics have been analyzed through the development of an AMESim virtual test bench which aimed at simulating the frictional torques and the leakage flow rates in accordance to experimental data. The purpose has been to insert the experimental curves directly in the AMESim model so that it is made as much as lookup tables. Specific mechanical and hydraulic submodels of the AMESim libraries have been identified and simulation tests have been performed in order to select the more adapted ones to reproduce the friction and leakage effects. Figure 4 shows the virtual prototype which simulates the vane pump performance using a generic implementation of its energy losses. At hydraulic ports 1 and 2, the line pressure is the input signal while at port 3 the input signal is represented by motor torque. 


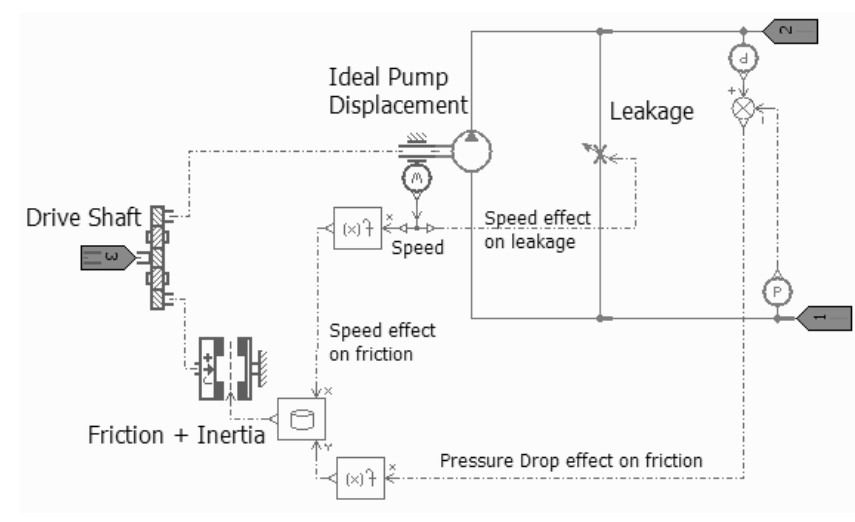

Figure 4: Virtual prototype of vane pump

The effective frictional torques and the leakage flow rates of the vane pump, are expressible through non-linear functions of operating conditions: pressure drop, pump speed and fluid temperature. The fluid temperature influences both frictional torques and leakages by means of variations of the dynamic viscosity of hydraulic fluid. At this modeling level and as a first step, it was assumed that:

- the operating temperatures are maintained constant. So the dependence to viscosity is not taken into account and postponed to next modeling level

Therefore the frictional torques and leakages are considered dependent only on hydraulic lines pressure drop and on the pump speed. The frictional torque is expressed in general form by eq. (30):

$$
T_{f p}=f_{f}(\Delta p, \omega)
$$

while the leakage flow rates got from eq. (31):

$$
Q_{l p}=f_{l}(\Delta p, \omega)
$$

In order to simulate the frictional torques in the form of the eq. (30), the component FR1RK000 in the mechanical library of AMESim has been selected (fig. 5) for its ability to vary friction versus operating conditions. It is a rotary friction torque generator based on Karnopp model that deals and solves efficiently the numerical implementation of transition between sticking and sliding conditions [17]. As required by the Karnopp model, the motor-pump rotor inertia is included in the friction component.

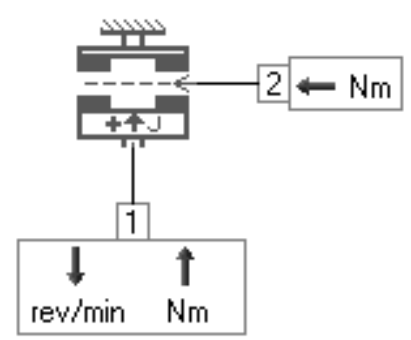

Figure 5: Component for pump frictions simulation, FRIRK000, from [18]
The experimental data of frictions torques, implemented in AMESim by means of an ASCII file in the format multi 1D tables [18], represent the input signal at port 2 of the component in fig. 5 and they permit to produce the desired friction versus pressure, velocity and even later temperature. A comparison between results provided by component and experimental curves has been carried out in order to verify the quality of the simulation (fig. 6).

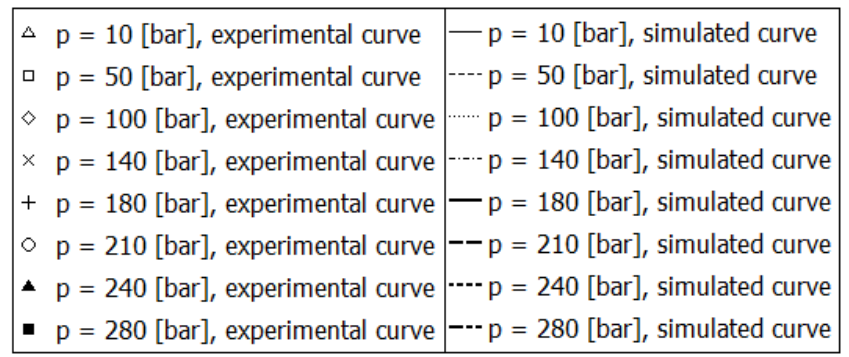

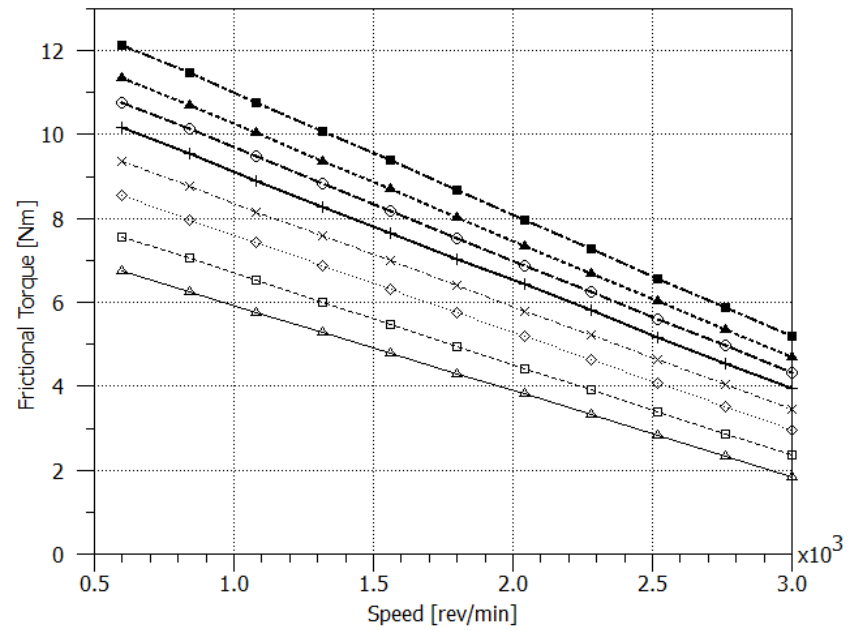

Figure 6: Frictional pump torques: experimental curves and curves obtained by AMESim model@45 ${ }^{\circ} \mathrm{C}$

Figure 6 shows that the simulation results agree well with experimental data inside the range of operating conditions performed in laboratory. In order to extend the range of operating conditions where the AMESim model is capable to provide values of frictional torques, an extrapolation of data has been proposed. A linear extrapolation has been carried out by the component FRIRK000 in the range of pump speed from -3500 to $3500 \mathrm{rev} / \mathrm{min}$ and for positive pressure drop values. Figure 7 shows both the experimental curves in the range of laboratory tests and the extrapolated curves.

It is interesting to mention that at zero speed the frictional torques are not null but they correspond to stiction. This would not have been possible if using tanh models. However, the Karnopp model requires defining a region around the exact condition of zero speed, where a threshold speed value is provided by user for transition between sticking and sliding conditions. In this context the value entered is of $0,36 \mathrm{rev} / \mathrm{min}, 1 / 10000$ of the rated velocity. 


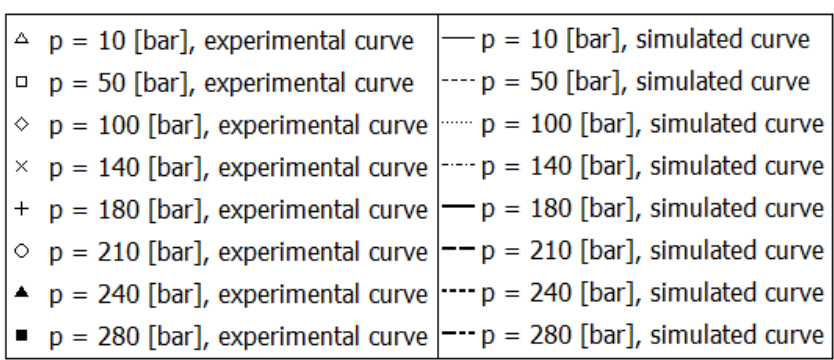

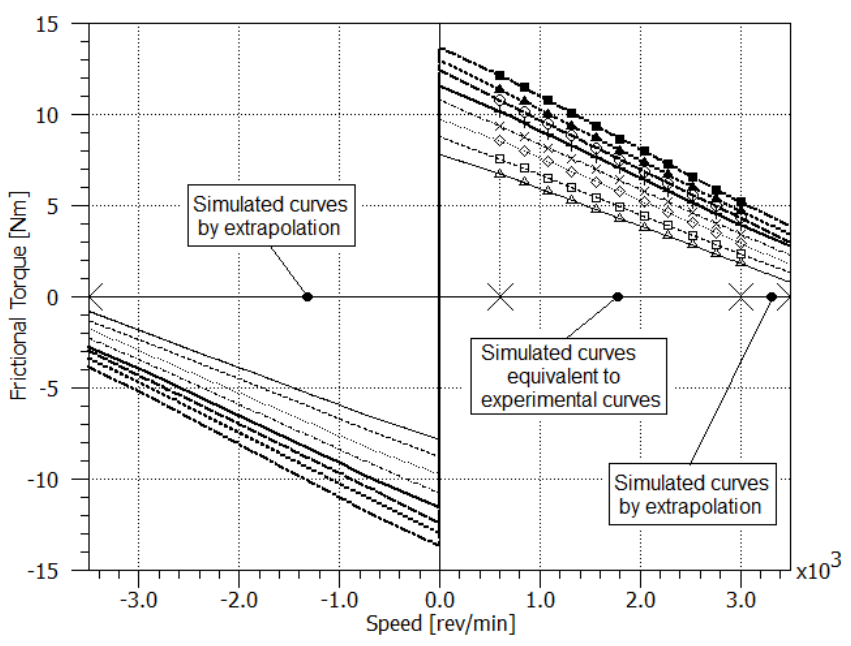

Figure 7: Frictional pump torques: extrapolation in the pump range: [-3500, 3500] rev/min provided by component FR1RK000

It is not easy to validate the extrapolated results provided at low speed near to zero through laboratory tests under constant operating temperature: in condition of low speed, the volumetric flow coming into pump is not sufficient to produce an adequate recirculation of fluid necessary to guarantee constant temperature inside pump. The AMESim model of frictional torques can be then improved by adding the dependence of friction to fluid temperature and by allowing testing the pump in those processes in which the evolution of temperature plays a fundamental role on performance degradations.

Concerning the leakages flow rates, the component VOR001 has been selected in the hydraulic AMESim library in order to simulate them by non-linear functions of pressure drop, pump speed (fig. 8) and later fluid temperature.

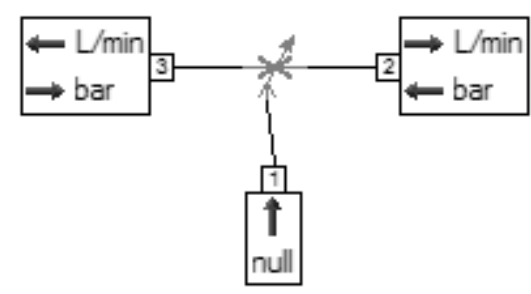

Figure 8: Component for pump leakages simulation, VOR001, from [18]
It represents a variable hydraulic orifice which provides, as outputs (ports 2 and 3 ), the leakage flow rate. In the component parameters table it is possible to insert directly the experimental data file. The signal at port 1 is the pump speed. Like for the frictional torques, the experimental data have been implemented in AMESim model. A comparison between results provided by component and experimental curves has been carried out in order to verify the quality of the simulation (fig. 9).

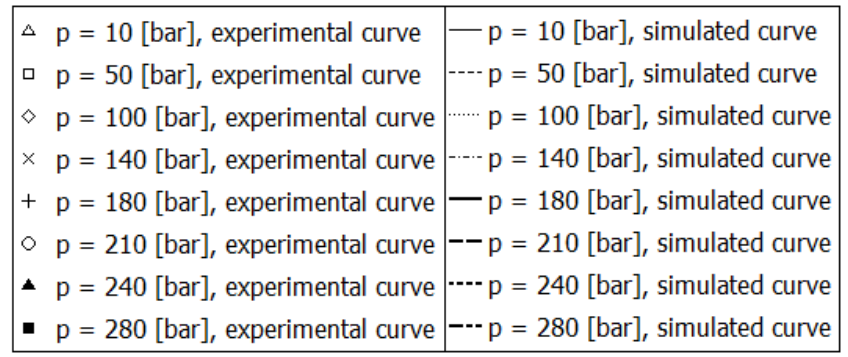

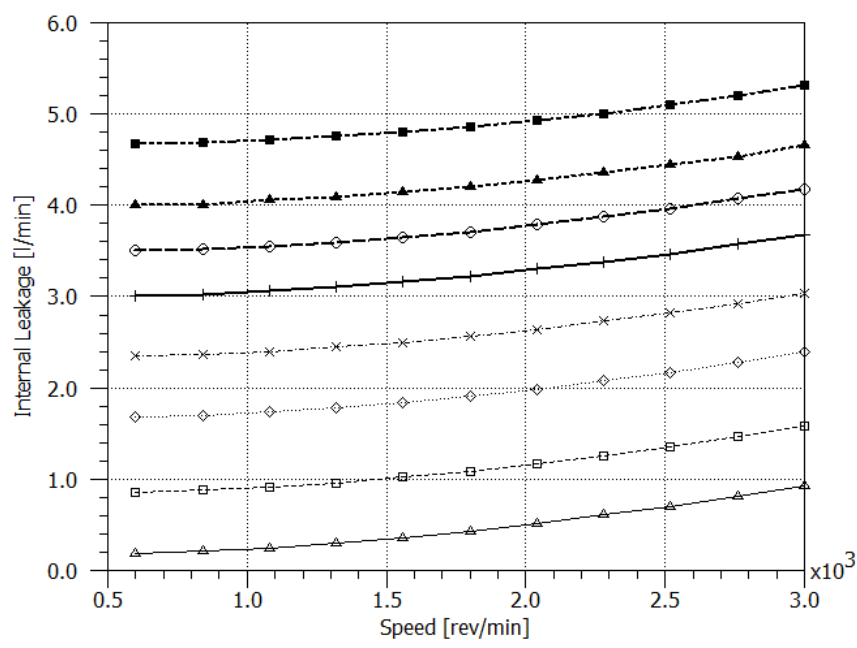

Figure 9: Internal pump leakages: experimental curves and curves obtained by AMESim model@45 ${ }^{\circ} \mathrm{C}$

Figure 9 shows that the simulation results agree well with experimental data inside the range of operating conditions performed in laboratory. In order to extend the range of operating conditions where the AMESim model is capable to provide values of leakage flow rates, an extrapolation of data has been proposed.

A linear extrapolation has been carried out by the component VOR001 in the range of pump speed from -3500 to $3500 \mathrm{rev} / \mathrm{min}$ and for positive pressure drop values. In addition, even if the vane pumps do not accept negative values of pressure drop because they are designed to operate in 2 quadrants only, the AMESim model enables reaching these conditions. Figure 10 shows both the experimental curves in the range of laboratory tests and the extrapolated curves. 


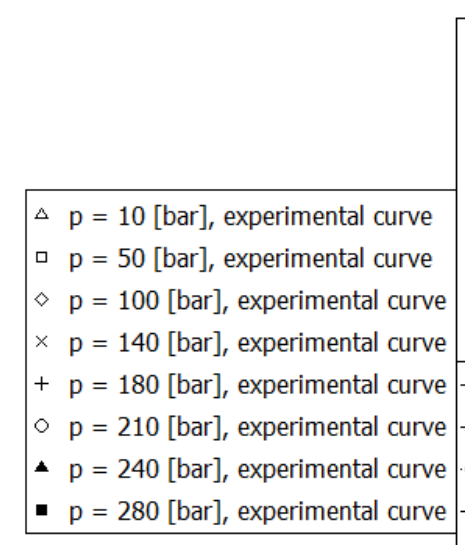

$-\mathrm{p}=10[\mathrm{bar}]$, simulated curve

--- $\mathrm{p}=50[\mathrm{bar}]$, simulated curve

..... $p=100[$ bar $]$, simulated curve

$\cdots p=140[$ bar $]$, simulated curve

$-\mathrm{p}=180[\mathrm{bar}]$, simulated curve

$--p=210[b a r]$, simulated curve

$\cdots p=240[\mathrm{bar}]$, simulated curve

$--p=280[$ bar $]$, simulated curve

- $p=-10[\mathrm{bar}]$, simulated curve

- $p=-50[\mathrm{bar}]$, simulated curve

๑. $\mathrm{p}=-100[\mathrm{bar}]$, simulated curve

$+\cdots p=-140[$ bar $]$, simulated curve

$* \mathrm{p}=-180[\mathrm{bar}]$, simulated curve

$\rightarrow p=-210[\mathrm{bar}]$, simulated curve

$-\because-p=-240[b a r]$, simulated curve

$\leftrightarrow-p=-280[\mathrm{bar}]$, simulated curve

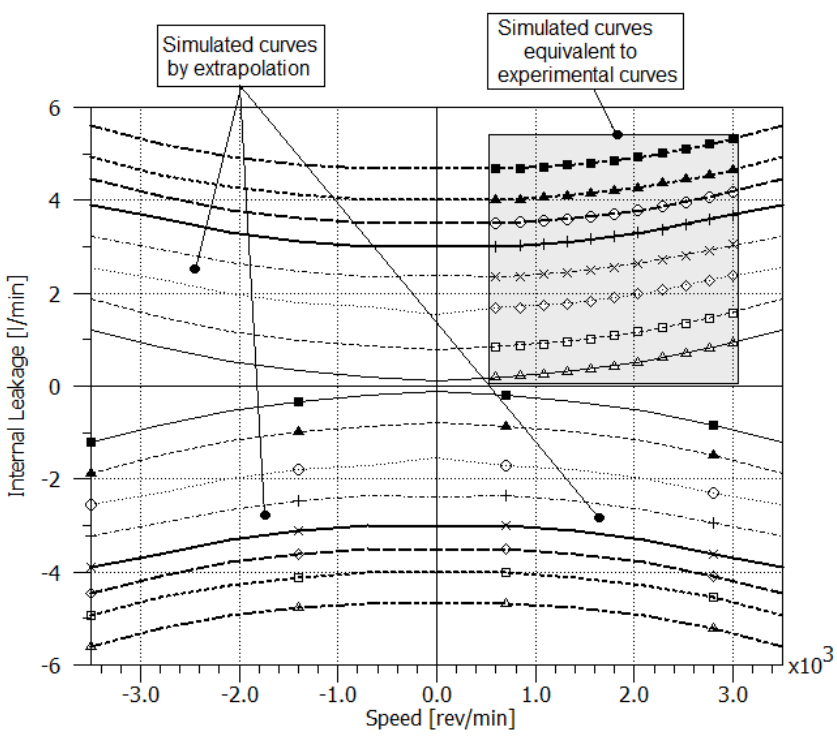

Figure 10: Internal pump leakages: extrapolation in the pump range: $[-3500,3500] \mathrm{rev} / \mathrm{min}$ provided by component VOR001

Due to difficulty to maintain constant the operating temperature during laboratory tests, as already described for frictional torques, it is not an easy task to validate the extrapolated results provided at low speed near to zero. The AMESim model of internal pump leakages can be then improved by adding the dependence to the fluid temperature.

\subsection{Parametric model of pump frictions and leakages}

A linear parametric representation model of energy losses has also been developed, capable to approximate the experimental curves of pump frictions and leakages, in accordance with the Linear Gap Theory for radial gap (LGT) [19]. This model represents a useful tool in the control unit design of the EHA module in order to assure system performance requirements.
The parametric model proposes a solution of the first degree polynomial type for both frictional torques and internal pump leakages:

$$
\begin{gathered}
T_{f p}=\alpha_{f p}+\beta_{f p} \omega+\gamma_{f p} \Delta p \\
Q_{l p}=\beta_{l p} \omega+\gamma_{l p} \Delta p
\end{gathered}
$$

where $\alpha_{i}, \beta_{i}$ and $\gamma_{i}$ are the parameters determined through the Least Square Method (LSM).

The LSM's objective is to find a good estimation of parameters, in order to fit the set of experimental data, by minimizing the sum of squared residuals (SSR). By varying the values of the parameters $\alpha_{i}, \beta_{i}$ and $\gamma_{i}$ of the eq.s (32) and (33), it has been possible to obtain the values of them which makes SSR minimum.

Figure 11 shows the comparison between experimental curves and parametric model curves of the frictional pump torques.

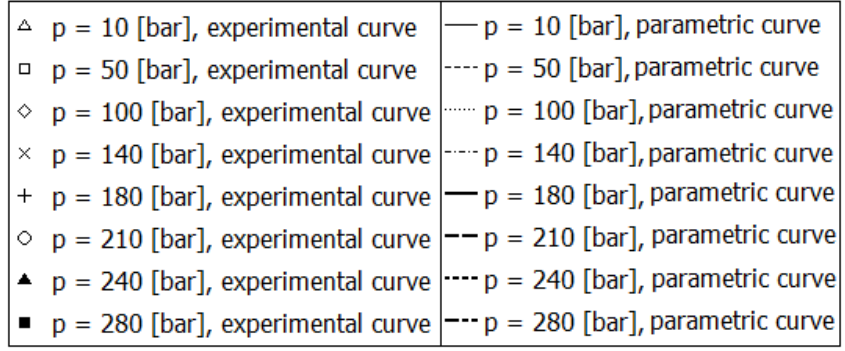

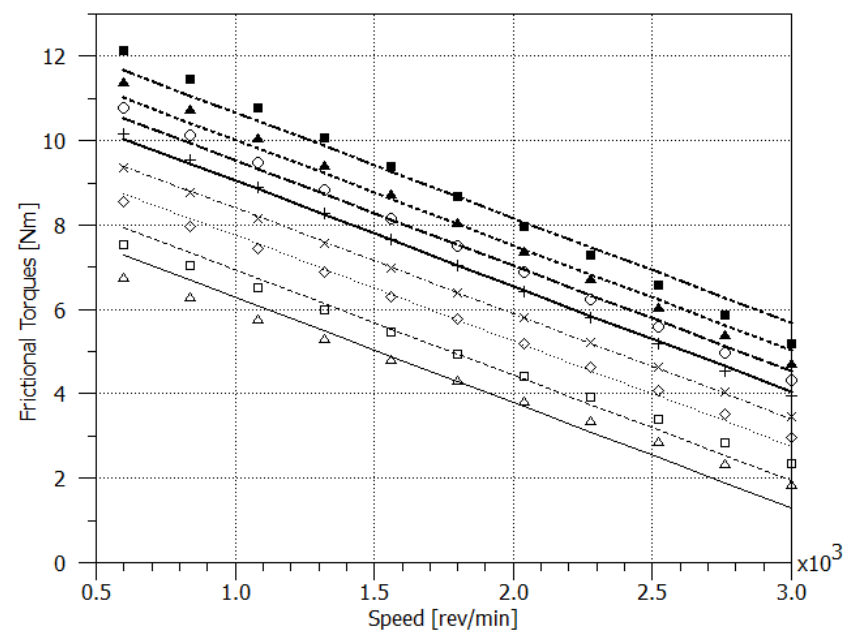

Figure 11: Frictional pump torques: experimental curves and curves obtained by parametric model@ $@ 45^{\circ} \mathrm{C}$

The relative error in percentage of frictional torques has been computed as:

$$
e_{\text {rel }}^{T} \%=\frac{\left(T_{f p}^{\text {experimental }}-T_{f p}^{\text {model }}\right)}{T_{f p}^{\text {experimental }}} 100
$$


It is possible to assert that the major region is characterized by an error $e_{r e l}^{T}$ less than $5 \%$ while only in a small region of high speed ( $>2600 \mathrm{rev} / \mathrm{min})$ and low pressure $(10 \mathrm{bar}<\Delta \mathrm{p}$ $<50 \mathrm{bar}$ ) the $e_{\text {rel }}^{T}$ overcomes $15 \%$ but never exceed $29 \%$.

Figure 12 shows the comparison between experimental curves and parametric model curves of the internal pump leakages.

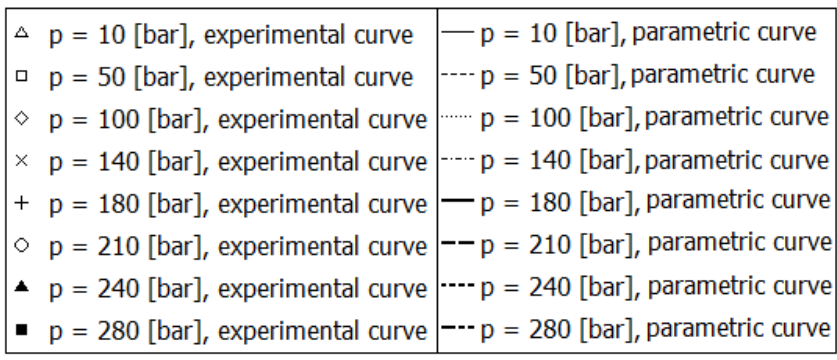

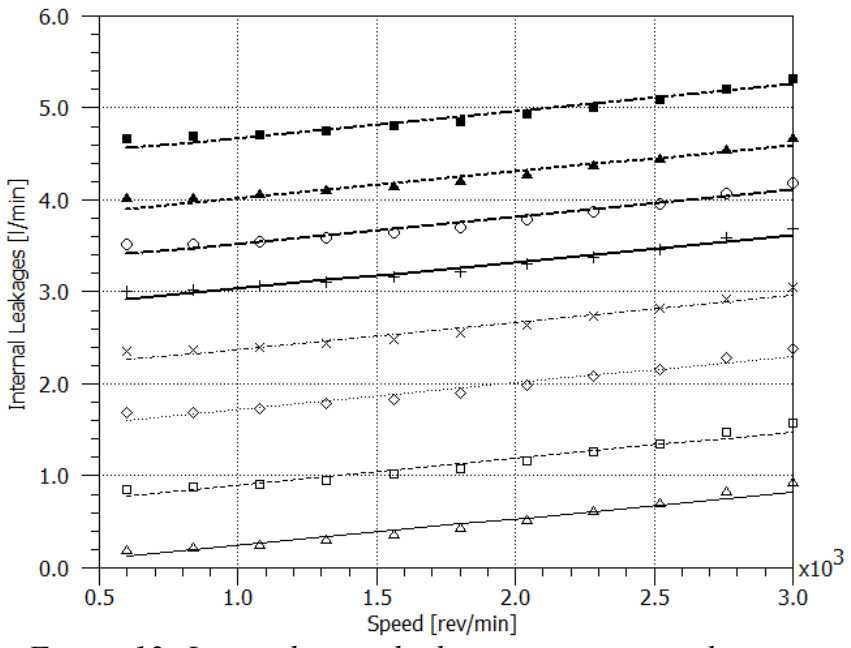

Figure 12: Internal pump leakages: experimental curves and curves obtained by parametric model@45 ${ }^{\circ} \mathrm{C}$

The relative error in percentage of internal leakages has been computed as:

$$
e_{\text {rel }}^{Q} \%=\frac{\left(Q_{l p}^{\text {experimental }}-Q_{l p}^{\text {model }}\right)}{Q_{l p}^{\text {experimental }}} 100
$$

Also in this case, the major region is characterized by $e_{r e l}^{Q}$ less than $5 \%$ while only in a small region of low speed $(<$ $800 \mathrm{rev} / \mathrm{min})$ and low pressure $(10 \mathrm{bar}<\Delta \mathrm{p}<50 \mathrm{bar})$ the error overcomes $15 \%$ but never exceed $29 \%$.

In conclusion, the parametric model based on the linear gap theory is able to reproduce the pump frictional torques and leakages experimental curves in the speed range [600, 3000] $\mathrm{rev} / \mathrm{min}$ and pressure drop range $[10,280]$ bar, with relative errors in percentage of experimental data under the $5 \%$ in the majority of operating conditions. In next analysis, it will be necessary to extend the model capability and check its validity in a wider range of speed by taking into account also starting pump conditions.

\section{Conclusion}

The paper has dealt with modeling and simulation at three level of accuracy of an EHA module for industrial applications. An EHA innovative solution has been showed that takes advantage of fixed-displacement vane pump and of closed-loop control respectively on: rod position, cylinder pressure and motor shaft speed. A preliminary analysis has been achieved by means of architectural model that has permitted to relate the steady-state operation with fundamental parameters to be defined. An initial approach to control synthesis has been exposed by providing the designer with key relationships between open loop statics and dynamics and fundamental parameters. The parasitic effects have been added and their influence on hydraulic and hydromechanical modes has been analyzed. In order to get a realistic representation of losses that play role for power sizing and temperature management, two types of models were implemented using standard components of the LMSAMESim libraries. Both frictional torque and internal leakage at vane pump level have been reproduced well using either look-up tables or parametric models of low complexity.

A complete virtual prototype, got by adding the detail model of the vane pump, exposed in this paper, and the models of other components constituting the EHA module, would represent an useful instrument to evaluate the energy consumption during a typical mission of injection molding machines and to optimize the EHA module design by simulating the influence of design choices on energy saving, closed loop performance and heat balance.

Another important aspect in the modelling of the EHA module to be taken into account is the thermo-hydraulic coupling: the system performances are hugely dependent on operating temperature because it strongly influences the hydraulic fluid properties. These effects become key design drivers in specific operating conditions, e.g. when pressure has to be accurately controlled without jack displacement phase of plastic injection. As the pump has no case drain to collect internal leakage and to take away heat coming from internal energy losses even a small error in modeling friction and leakage will alter the predictability of the EHA model and therefore not reproduce the potential issue of thermal divergence. For this reason, the ongoing work consists in improving accuracy of the EHA virtual prototype by developing thermal-hydraulic simulation at system level and generating expert tools for component selection from catalogues and controller setting.

\section{Nomenclature}

\begin{tabular}{|c|c|c|}
\hline Designation & Denotation & Unit \\
\hline$A_{c}$ & Section pipe area & {$\left[m^{2}\right]$} \\
\hline$a_{j}$ & $\begin{array}{l}\text { Jack cross-chamber leakage } \\
\text { coefficient }\end{array}$ & {$\left[\mathrm{m}^{4} \mathrm{~s} / \mathrm{kg}\right]$} \\
\hline
\end{tabular}




\begin{tabular}{|c|c|c|c|c|c|}
\hline \multirow{2}{*}{$a_{p}$} & \multirow{2}{*}{$\begin{array}{l}\text { Internal pump leakage } \\
\text { coefficient }\end{array}$} & \multirow{2}{*}[\mathrm{m}^{4}\mathrm{s}/\mathrm{kg}]{} & \multirow{2}{*}{$\begin{array}{l}t_{s} \\
U_{m}\end{array}$} & \multirow{2}{*}{$\begin{array}{l}\text { Response time } \\
\text { Motor voltage }\end{array}$} & \multirow{2}{*}{$\begin{array}{l}{[s]} \\
{[V]}\end{array}$} \\
\hline & & & & & \\
\hline$B$ & $\begin{array}{l}\text { Total viscous friction } \\
\text { coefficient }\end{array}$ & {$[\mathrm{Nm} \mathrm{s} / \mathrm{rad}]$} & $U_{s}$ & Supply voltage & {$[V]$} \\
\hline$B_{m}$ & $\begin{array}{l}\text { Motor viscous friction } \\
\text { coefficient }\end{array}$ & {$[\mathrm{Nm} \mathrm{s} / \mathrm{rad}]$} & $V$ & $\begin{array}{l}\text { Total volume of pipe and } \\
\text { jack chamber }\end{array}$ & {$\left[m^{3}\right]$} \\
\hline \multirow{2}{*}{$B_{p}$} & \multirow{2}{*}{$\begin{array}{l}\text { Pump viscous friction } \\
\text { coefficient }\end{array}$} & \multirow{2}{*}[\mathrm{Nm}\mathrm{s}/\mathrm{rad}]{} & $V_{c}$ & Conduit Volume & {$\left[m^{3}\right]$} \\
\hline & & & $V_{j}$ & Jack chamber volume & {$\left[m^{3}\right]$} \\
\hline$D_{c}$ & Pipe diameter & {$[m]$} & $V_{0}$ & Pump displacement & {$\left[\mathrm{m}^{3} / \mathrm{rad}\right]$} \\
\hline$F_{f j}$ & $\begin{array}{l}\text { Cylinder/piston frictional } \\
\text { force }\end{array}$ & {$[N]$} & $x$ & Rod position & {$[m]$} \\
\hline \multirow{2}{*}{$F_{j}$} & \multirow{2}{*}{ Jack force } & \multirow{2}{*}[N]{} & $\dot{x}$ & Rod speed & {$[\mathrm{m} / \mathrm{s}]$} \\
\hline & & & $\ddot{x}$ & Rod acceleration & {$\left[\mathrm{m} / \mathrm{s}^{2}\right]$} \\
\hline$f$ & $\begin{array}{l}\text { Jack viscous friction } \\
\text { coefficient }\end{array}$ & {$[\mathrm{kg} / \mathrm{s}]$} & $\alpha$ & Power modulation factor & {$[-]$} \\
\hline$f_{h}$ & Hydraulic natural frequency & {$[\mathrm{Hz}]$} & $\alpha_{f p}$ & Static friction coefficient & {$[\mathrm{Nm}]$} \\
\hline \multirow{2}{*}{$f_{h m}$} & \multirow{2}{*}{$\begin{array}{l}\text { Hydromechanical natural } \\
\text { frequency }\end{array}$} & \multirow{2}{*}[\mathrm{Hz}]{} & $\alpha_{k}$ & Stick-slip coefficient & {$[\mathrm{rad} / \mathrm{s}]$} \\
\hline & & & $\beta$ & Bulk modulus & {$[P a]$} \\
\hline$J$ & \multirow{2}{*}{$\begin{array}{l}\text { Total inertia } \\
\text { Motor inertia }\end{array}$} & \multirow{2}{*}{$\begin{array}{l}{\left[\mathrm{kg} \mathrm{m}^{2}\right]} \\
{\left[\mathrm{kg} \mathrm{m}^{2}\right]}\end{array}$} & $\beta_{f p}$ & Viscous friction coefficient & {$[\mathrm{Nm} \mathrm{s} / \mathrm{rad}]$} \\
\hline$J_{m}$ & & & $\beta_{l p}$ & Speed coefficient for leakage & {$\left[\mathrm{m}^{3} / \mathrm{rad}\right]$} \\
\hline$J_{p}$ & Pump inertia & {$\left[\mathrm{kg} \mathrm{m}^{2}\right]$} & & Pressure coefficient for & \\
\hline$K_{m}$ & $\begin{array}{l}\text { Back-electromotive force } \\
\text { coefficient }\end{array}$ & {$[N m / A]$} & $\gamma_{f p}$ & friction & {$\left[m^{3}\right]$} \\
\hline$I_{m}$ & coefficient & {$[A]$} & $\gamma_{l p}$ & $\begin{array}{l}\text { Pressure Coefficient for } \\
\text { leakage }\end{array}$ & {$\left[\mathrm{m}^{4} \mathrm{~s} / \mathrm{kg}\right]$} \\
\hline$I_{s}$ & Supply current & {$[A]$} & $\Delta p$ & Cylinder pressure & {$[P a]$} \\
\hline$L$ & Motor inductance & {$[H]$} & $\Delta p_{c}$ & Pipe pressure drop & {$[\mathrm{Pa}]$} \\
\hline$L_{c}$ & Pipe length & {$[m]$} & $\Delta \dot{p}$ & $\begin{array}{l}\text { Cylinder pressure variation in } \\
\text { time }\end{array}$ & {$[\mathrm{Pa} / \mathrm{s}]$} \\
\hline$M$ & Total mass & {$[\mathrm{kg}]$} & & & \\
\hline$Q_{j}$ & Input jack flow rate & {$\left[m^{3} / s\right]$} & $\lambda$ & Friction factor & {$[-]$} \\
\hline$Q_{l j}$ & Jack chamber leakage & {$\left[m^{3} / s\right]$} & $\rho$ & Fluid density & {$\left[\mathrm{kg} / \mathrm{m}^{3}\right]$} \\
\hline$Q_{l p}$ & Internal pump leakage & {$\left[m^{3} / s\right]$} & $\omega$ & Hydraulic undamned natura & $\lfloor\mathrm{rad} / \mathrm{s}\rfloor$ \\
\hline$Q_{p}$ & Output pump flow rate & {$\left[\mathrm{m}^{3} / \mathrm{s}\right]$} & $\omega_{h}$ & pulsation & {$[\mathrm{rad} / \mathrm{s}]$} \\
\hline$R$ & Motor resistance & {$[\mathrm{Ohm}]$} & $\omega_{h m}$ & $\begin{array}{l}\text { Hydromechanical undamped } \\
\text { natural nulsation }\end{array}$ & {$[\mathrm{rad} / \mathrm{s}]$} \\
\hline$R e$ & Reynolds number & {$[-]$} & & & \\
\hline$S$ & Active area of piston & {$\left[m^{2}\right]$} & $\dot{\omega}$ & Motor shaft acceleration & {$\left[\mathrm{rad} / \mathrm{s}^{2}\right]$} \\
\hline$T_{c}$ & Coulomb torque & {$[\mathrm{Nm}]$} & $\xi_{h}$ & Hydraulic damping ratio & {$[-]$} \\
\hline$T_{f m}$ & Frictional motor torque & {$[\mathrm{Nm}]$} & $\xi_{h m}$ & $\begin{array}{l}\text { Hydromechanical damping } \\
\text { ratio }\end{array}$ & {$[-]$} \\
\hline
\end{tabular}

\section{References}

[1] J-C Maré. Towards more electric drives for embedded applications: (re)discovering the advantages of hydraulics. $7^{\text {th }}$ International Fluid Power Conference, Aachen, Germany, 2010. 
[2] S Helduser. Development trends in electrohydraulic drives and controls. $6^{\text {th }}$ International Fluid Power Conference, Dresden, Germany, 2008.

[3] K Muller, and U Dorn. Variable speed drives customer benefits in injection molding machines and presses. $7^{\text {th }}$ International Fluid Power Conference, Aachen, Germany, 2010.

[4] S Helduser. Improved energy efficiency in plastic injection moulding machines. $8^{\text {th }}$ Scandinavian International Conference of Fluid Power, Tampere, Finland, 2003.

[5] J-C Maré. Combining hydraulics and electrics for innovation and performance improvement in aerospace actuation. $12^{\text {th }}$ Scandinavian International Conference of Fluid Power, Tampere, Finland, 2011.

[6] A Feuser. Modern electrohydraulic drive technology for stationary machinery in industrial automation. $7^{\text {th }}$ International Fluid Power Conference, Aachen, Germany, 2010.

[7] K R McCullough. Design and characterization of a dual electro-hydrostatic actuator. Department of Mechanical Engineering, McMaster University, Hamilton, Canada, 2011.

[8] S Habibi, and A Goldenburg. Design of a new high performance electrohydraulic actuator. IEEE/ASME Transactions on Mechatronics, Vol.5, 2000.

[9] Parker Catalogue. Compact electro-hydraulic actuator (EHA). Bulletin HY22-3200A/US

[10]D van den Bossche. The A380 flight control electrohydrostatic actuators, achievements and lessons learnt. $25^{\text {th }}$ International Congress of the Aeronautical Sciences, Hamburg, Germany, 2006.

[11] A E Fitzgerald, C Jr Kingsley, and S D Umans. Electric Machinery. $6^{\text {th }}$ edition, McGraw Hill, 2003.

[12]H E Merritt. Hydraulic Control Systems. John Wileys and Sons, Cincinnati, Ohio, 1967. ISBN 0-471-59617-5.

[13] J-C Maré. Actionneurs hydrauliques - commande. Article S7531, série Mesure et Régulation, Encyclopédie des Techniques de l'Ingénieur, page 17, Septembre 2002.

[14]F H Raven. Automatic Control Engineering. $5^{\text {th }}$ edition, McGraw Hill, 1995.

[15] J-C Maré. Considerations pratiques sur les correcteurs PID dans le domaine de l'énergie fluide. Revue Fluides, pp 8-17, Numéro Spécial Matériels, Septembre 2000
[16] E G Papadopoulos, and G C Chasparis. Analysis and model-based control of servomechanisms with friction. Proceedings of the IEEE/RSJ International Conference on Intelligent Robots and System, Lausnne, Switzerland, 2002.

[17]D Karnopp. Computer simulation of stick-slip friction in mechanical dynamic systems. Journal of Dynamic Systems, Measurement and Control. Vol. 7, March 1985.

[18]LMS-AMESim. AMEHelp

[19]P Krus. Formula Book For Hydraulics and Pneumatics. Fluid and Mechanical Engineering Systems, Department of Management and Engineering, Linkoping University, Sweden. Revised 2008-10-27. 